\title{
Diagnostic efficacy of sepsis-screen parameters (individual and combined) compared to blood culture and clinico-etiological profile of neonatal sepsis in preterm newborns
}

\author{
Beck P. ${ }^{1}$, Rathia S.K. ${ }^{2}$, Phuljhele S. ${ }^{3}$, Kurrey S. ${ }^{4}$ \\ ${ }^{1}$ Dr. Pratima Beck, Associate Professor, ${ }^{2}$ Dr. Santosh Kumar Rathia, Assistant Professor, ${ }^{3}$ Dr. Sharja Phuljhele, Professor \\ $\&$ HOD, ${ }^{4}$ Dr. Swati Kurrey, Postgraduate student; above all authors are affiliated to Department of Paediatrics, Pt. J.N.M. \\ Medical College, Raipur, Chhattisgarh, India.
}

Corresponding Author: Dr. Santosh Kumar Rathia, MIG (Deluxe)-217, Phase-2, Kabirnagar, Near Guru Gobind Chowk, Raipur, 492099, Chhattisgarh, India. E-mail: drsantoshrathia84@gmail.com

\begin{abstract}
Introduction: Sepsis alone or along with other morbidity is the major contributor to mortality in both preterm and term babies accounting for more than a third of neonatal deaths in developing countries. Overall incidence of early onset sepsis (EOS) is higher in preterms and very low birth weight babies. Sepsis and related mortality is largely preventable with rational antimicrobials plus supportive therapy after timely detection of clinical sepsis, risks, complications, and with help of early screening laboratory-markers. Material and Method: This was a prospective, observational, hospitalbased study to compare the efficacy between various sepsis-screening tests \& blood culture in early diagnosis of neonatal sepsis in preterms, conducted in NICU at Pt. JNM Medical College, Raipur, conducted during April 2018-September 2018. By using statistical Sample-size formula, 125 preterm neonates were enrolled. Detailed history and clinical findings were recorded on a predesigned proforma. All five sepsis-screen tests were done within 24 hours of birth or at clinical presentation before starting antibiotics, for all preterms at risk of EOS (based on neonatal/maternal risk factors $>3$ ) and/or developing clinical sepsis. Data was compiled, tabulated and analyzed using Microsoft-SPSS version20. Result: Out of 125 patients, all were preterm $<37$ weeks by gestation $(<28$ week=3.2\%, 28-32 weeks=52\%, $32-35$ weeks $=33.6 \%$ and late preterm: $35-37$ weeks $=11.2 \%$ ). Birth weight wise, $15.2 \%$ were ELBW, 45.6\% were VLBW, and $39.2 \%$ weighed $>1500 \mathrm{~g}$. Out of all sepsis-suspect and at risk cases, EOS risk factors $(>/=3$ of maternal plus neonatal risks) was present in $47.2 \%$, while EOS was suspected at admission in $83.2 \%$ cases on clinical ground. Overall sepsis screening was positive ( $>=2$ parameters) in $40 \%$ of all subjects [ $24 \%$ having only $2,10.4 \%$ having 3 and $5.6 \%$ having 4 tests positive], though $>50 \%$ cases had single test positive (considered screen-ve). Although $>88 \%$ cases developed clinical sepsis (EOS+LOS), only 40\% were detected by screen positive (as probable sepsis), and 35.2\% were blood culture positive (proven sepsis). Conclusion: The combinations of sepsis makers yielded better diagnostic results than single tests and proved to be a valuable aid for early diagnosis of neonatal sepsis in preterms along with blood culturesensitivity.
\end{abstract}

Keywords: Preterm, Neonatal sepsis, EOS, Risk factors, Sepsis screen parameters, Blood culture

\section{Introduction}

Neonatal sepsis alone and/or severe sepsis resulting into multiple vital organ dysfunctions contributes to $30-50 \%$ of neonatal deaths in developing countries; while up to $20 \%$ of neonates develop sepsis and approximately $1 \%$ die of sepsis or related causes $[1,2]$. Neonatal sepsis, clinical onset wise, may present as early (EOS, within

Manuscript received: $8^{\text {th }}$ July 2019

Reviewed: $18^{\text {th }}$ July 2019

Author Corrected: $26^{\text {th }}$ July 2019

Accepted for Publication: $31^{\text {st }}$ July 2019
$72 \mathrm{hrs}$ ) or late (LOS, after $72 \mathrm{hrs}$ ). Although, overall incidence of EOS is 1-2 per 1000 live births, while it's twice in preterms (with or without LBW), and even more (15-23/1000) in ELBW babies <1000g; LOS also majorly affects VLBW neonates having more susceptibility to post-natal infections [3,4]. The inability of early neonates, especially preterm ones, to produce the adequate inflammatory response makes them more susceptible to bacterial invasion of the blood stream in 


\section{Original Research Article}

comparison to older children and adults [5]. However, since, early features of neonatal septicaemia are often non-specific; distinguishing between septic and nonseptic babies may not always be easy and indiscriminate use of antibiotics (for all presumed bacterial sepsis) may lead to emergence of catastrophically resistant bugs [6]. Although a positive blood culture still remains "the gold standard" for diagnosing sepsis. Moreover, micro-biological culture facilities in many developing countries are still inadequate and results take atleast 4872 hours, resulting in treatment delays [7, 8]. Most paediatricians, therefore have to rely, even today, on a sepsis screen (includes various haematological and biochemical markers) for a quick and reliable diagnosis.

However, the reported sensitivity and specificity of these individual markers is rather low and some of the tests are labour intensive or require a highly trained technician to produce an accurate result [6]. Furthermore, the greatest predictability usually results from not a single assay, but a combination of assays[9]. The present study was aimed at comparing efficacy of various sepsis-screen markers, individually and in

\section{Material and Methods}

Study setting: Neonatal ICU of Dr. BRAM Hospital, and Pt. JNMMC, Raipur, Chhattisgarh.

Study design: Cross sectional, observational study with comparison of diagnostic accuracy.

Study period: Six months for data collection (March 2018 to August 2018).

Sample size:125 preterm newborns admitted during the study period with 'suspected/presumed sepsis' or evenjust 'risk of sepsis'(early onset sepsis) were included in this study.

Inclusion criteria: All preterm newborns admitted to NICU during study period with suspected sepsis or at risk of sepsis (especially EOS) were enrolled after proper history taking and through clinical examination.

\{The NNF accepted definition of 'neonatal sepsis' as a clinical syndrome included- poor feeding, irritability / excessive cry, lethargy, poor cry and reflexes, fever, hypothermia, jaundice, vomiting, abdominal distension, tachypnea and grunting, convulsions, diarrhoea, pustules, sclerema, cyanosis, bulging fontanelle, DIC/bleeding, poor perfusion / shock, apnea\}.

Major risk factors of sepsis/EOS included in predictive perinatal scoring as per NNF criteria [10] were- LBW/Preterm newborn, maternal fever, foul smelling liquor, PROM $>18 \mathrm{hrs,} \mathrm{prolonged} \mathrm{labour,} \mathrm{unclean} \mathrm{or}>3$ vaginal examinations, perinatal asphyxia (Apgar $<4$ at 1 min.)

Exclusion criteria: Term babies with $>37$ week GA, major congenital anomalies of GI system, respiratory system, cardiovascular system, central nervous system, suspected inborn errors of metabolism.

Study methodology and data collection: Relevant laboratory investigations were done; for all preterms with risk factors or suspected features of sepsis; even for the newborn has yet not developed clinical sign/symptoms of sepsis. Preferably sample for all test of sepsis screen and blood culture were taken within 24 hrs of birth or at admission for suspected sepsis, before starting IV antibiotics. To ascertain demographic details, relevant maternal history and to note the findings of clinical examinations of neonates, a well designed structural Performa was used where all requisite details were filled up pertaining to the study. The sepsis screen parameters were taken as per latest available NNF guidelines (2010) and even old standard references [11-14]. If any two of the following parameters (Table-1) are positive or significant, the sepsis screen is considered positive.

Pediatric Review: International Journal of Pediatric Research Available online at: www.medresearch.in 373|P a g e 
Table-1: Sepsis- screen parameters.

\begin{tabular}{|l|l|}
\hline Components & Abnormal value \\
\hline a) TLC (Total leukocyte count) & $<5000$ cells $/ \mathrm{mm} 3$ \\
\hline b) IT-Ratio (Immature: Total PMN cells) & $>0.2$ \\
\hline c) ANC (Absolute Neutrophil Count) & $<1800$ cells $/ \mathrm{mm} 3$ \\
\hline d) m-ESR (micro-ESR) & $>15 \mathrm{~mm}$ at 1 hour \\
\hline e) CRP (C reactive protein) & $>1 \mathrm{mg} / \mathrm{dl}$ \\
\hline
\end{tabular}

The ANC cut off was kept $<1800$ cells/mm3 even for preterm subjects (with/without VLBW), for uniformity on analysing results for patients enrolled and sampled at different age while admitted due to large proportion of extramural and referred cases.

Statistical analysis: All the study parameters were entered in the excel sheet and were analysed using SPSS-20 software. Descriptive parameters were used for the univariate analysis and expressed in frequency and percentage. Sensitivity, specificity, NPV and PPV of septic screen were calculated for each individual laboratory marker as well as combination of two markers.

\section{Results}

Demographic characteristics of study subjects- In this study 125 preterm newborns suspected to have sepsis or risk of early onset sepsis were enrolled, of which majority were male (94.4\%) and 58.4\% were out-born/extramural admission in the study setting. Among these preterm subjects, birth weight wise, 15.2\% were ELBW $(<1000 \mathrm{~g}), 45.6 \%$ were VLBW, and $39.2 \%$ had LBW weighing 1500-2500g.Although by gestational age, all study subjects were preterms $(<37$ weeks), majority (55.2\%) had extreme prematurity ( $<32$ week, including 3\% with GA<28wks), 33.6\% between $32-35$ weeks and only $11.2 \%$ were so called 'late preterms' (35-37 week).With respect to clinical onset of sepsis, vast majority of presumed/suspected sepsis (83.2\% of cases) had EOS (early onset within 72 hours) as shown in table 2.

Table-2: Demographic data for study subjects.

\begin{tabular}{|c|c|c|}
\hline Characteristics & Frequency & Percentage \\
\hline \multicolumn{3}{|l|}{ Sex wise distribution } \\
\hline Female & 7 & 5.6 \\
\hline Male & 118 & 94.4 \\
\hline \multicolumn{3}{|l|}{ Birth weight } \\
\hline Less than $1000 \mathrm{~g}$ & 19 & 15.2 \\
\hline $1000-1500 \mathrm{~g}$ & 57 & 45.6 \\
\hline $1500-2500 \mathrm{~g}$ & 49 & 39.2 \\
\hline \multicolumn{3}{|l|}{ Gestational age } \\
\hline$<28$ weeks & 4 & 3.2 \\
\hline 28-32 weeks & 65 & 52 \\
\hline $32-35$ weeks & 42 & 33.6 \\
\hline 35-37 weeks & 14 & 11.2 \\
\hline \multicolumn{3}{|l|}{ Place of delivery } \\
\hline Inborn & 52 & 41.6 \\
\hline Outborn & 73 & 58.4 \\
\hline \multicolumn{3}{|l|}{ Age at onset (of sepsis) } \\
\hline Early onset sepsis & 104 & 83.2 \\
\hline Late onset sepsis & 21 & 16.8 \\
\hline
\end{tabular}




\section{Original Research Article}

Risk factors of neonatal sepsis amongst study subjects- Maternal risk factors for early onset sepsis amongst all presumed EOS or at risk cases (Table-3) shows that most of mothers $(51.2 \%)$ had risks due to $>3$ clean vaginal examination (mostly referred cases after multiple handling) and/or occasional single unclean vaginal examination, $36.8 \%$ had foul smelling liquor, $32 \%$ had intrapartum fever $>37.5$ degree Celsius, $21.6 \%$ had PROM $>18$ hours, $4 \%$ of newborns had documented perinatal asphyxia and $3.2 \%$ had meconium aspiration.

Table-3: Maternal Risk factors for early onset neonatal sepsis

\begin{tabular}{|c|c|c|}
\hline Maternal factors for sepsis & Frequency & Percentage \\
\hline Foul smelling liquor & 46 & 36.8 \\
\hline 1 unclean vaginal or $>3$ clean vaginal examination & 64 & 51.2 \\
\hline PROM $>18$ hrs & 27 & 21.6 \\
\hline Aspiration of meconium & 4 & 3.2 \\
\hline Intrapartum fever $>37.5$ & 40 & 32 \\
\hline Prolonged labour (sum of 1 st and 2 nd stage $>24 \mathrm{hrs}$ ) & 3 & 2.4 \\
\hline Perinatal asphyxia (Apgar score $<4$ at $1 \mathrm{~min}$ ) & 5 & 4 \\
\hline
\end{tabular}

Clinical presentation of preterm newborns with suspected sepsis- Amongst preterm subjects who presented with clinical features suspicious of sepsis (whether EOS or LOS), there were varied presenting complaints (see Table-4), most common being poor sucking/feeding (48\%), tachypnea (46\%), lethargy (41\%), irritability (26\%), grunting (22.4\%) and vomiting (18\%). Among less common initial/presenting findings in preterm babies were bleeding, petechiae, hypothermia and mottling/prolonged CRT.

Table-4: Presenting clinical symptoms/signs in preterm newborns with suspected sepsis

\begin{tabular}{|l|c|c|}
\hline Symptom & Frequency & Percent \\
\hline Poor feeding/ poor sucking & 60 & 48 \\
\hline Tachypnea & 57 & 45.6 \\
\hline Lethargy & 51 & 40.8 \\
\hline Irritability & 33 & 26.4 \\
\hline Grunting & 28 & 22.4 \\
\hline Vomiting & 22 & 17.6 \\
\hline Abdominal distension & 11 & 8.8 \\
\hline Hypothermia & 6 & 4.8 \\
\hline Bleeding & 5 & 4.8 \\
\hline Prolonged CRT or mottling & 4 & 3.2 \\
\hline Petechiae \& rash & 1 & 0.8 \\
\hline
\end{tabular}

Sepsis screen test results and interpretation- First sepsis-screen results of 125 preterm study subjects revealed (as in table-5) that individual tests had variable but low positivity with the increasing order of - ANC $(16 \%)>$ TLC $(19 \%)>$ CRP $(31 \%)>$ IT Ratio $(42 \%)>\operatorname{mESR}(45.6 \%)$.

Table-5: Initial sepsis-screening results amongst study subjects.

\begin{tabular}{|l|c|c|}
\hline Investigations/SS markers & Cut Off Values & Percent \\
\hline \multirow{2}{*}{ TLC } & $\mathbf{2 5 0 0 0}$ & $\mathbf{1 9 . 2}$ \\
\cline { 2 - 3 } & $>5000$ & 80.8 \\
\hline \multirow{2}{*}{ ANC } & $<\mathbf{1 8 0 0}$ & $\mathbf{1 6}$ \\
\cline { 2 - 3 } & $>1800$ & 84 \\
\hline \multirow{2}{*}{ CRP } & $>\mathbf{m g} / \mathbf{d l}$ & $\mathbf{3 1 . 2}$ \\
\cline { 2 - 3 } & $<1 \mathrm{mg} / \mathrm{dl}$ & 68.8 \\
\hline \multirow{2}{*}{ IT RATIO } & $>\mathbf{0 . 2}$ & $\mathbf{4 2 . 4}$ \\
\cline { 2 - 3 } & $<0.2$ & 57.6 \\
\cline { 2 - 3 } mESR & $>\mathbf{1 5 m}$ at $\mathbf{1 h o u r}$ & $\mathbf{4 5 . 6}$ \\
\cline { 2 - 3 } & $<15 \mathrm{~mm}$ & 55.2 \\
\hline
\end{tabular}




\section{Original Research Article}

All positive results of sepsis-screen parameters were analysed for single and combined positivity amongst study subjects (see table 6) and it was observed that around $10 \%$ cases had no positive result test out of 5 tests, $50 \%$ had single test positive, and significantly positive sepsis-screen result with $\geq 2+v e$ parameters was observed in $40 \%$ of all preterms with risk or clinical sepsis.

Table-6: Sepsis screen parameter positivity: individual and in combinations.

\begin{tabular}{|c|c|c|}
\hline $\begin{array}{c}\text { Total +veseptic-screen } \\
\text { parameters }\end{array}$ & Frequency of study subjects & Percentage \\
\hline 1 & 63 & 50.4 \\
\hline 2 & 30 & 24 \\
\hline 3 & 13 & 10.4 \\
\hline 4 & 7 & 5.6 \\
\hline Total +ve & $\mathbf{1 1 3}$ & $\mathbf{9 0 . 4}$ \\
\hline 0 (all test results -ve) & 12 & 9.6 \\
\hline Total subjects screened & 125 & 100 \\
\hline
\end{tabular}

Diagnostic predictability of sepsis-screen: probable sepsis vsculture-proven sepsis- For confirmed diagnosis of neonatal sepsis in all of our preterm subjects, blood culture was sent preferably before starting antibiotics. Out of 125 preterm newborns, 44 cases (35.2\%) had blood culture positive results indicating 'culture-proven sepsis' as all enrolled cases were suspected to have sepsis or had risk of developing it. Thus, in comparison to $35 \%$ confirmatory blood-culture results (proven sepsis), a proportionate $40 \%$ screen positive results suggest good diagnostic efficacy of standard sepsisscreening with combined tests including any of 2 parameters to define clinically suspected cases as 'probable sepsis'.(see Table 7)

Table-7: 'Probable sepsis'(SS +ve) versus blood-culture 'proven sepsis'

\begin{tabular}{|c|c|c|}
\hline Diagnostic certainty of sepsis & Frequency (N) & Percent \\
\hline Culture-proven sepsis & 44 & $\mathbf{3 5 . 2} \%$ \\
\hline Probable sepsis (SS+ve $\geq 2$ tests) & 50 & $\mathbf{4 0 \%}$ \\
\hline
\end{tabular}

Final patient outcome in this study setting- Final treatment outcome of preterms admitted with presumed sepsis or its risk revealed that only $43.2 \%$ patients were discharged and overall poor outcome in the study setting, with $31.2 \%$ deaths along with $25.6 \%$ taken LAMA (most of them being critical at that time), might be due to prematurity and other related co-morbidities.

\section{Comparison of diagnostic efficacy of various sepsis-screen (SS) markers}

Individual markers: Comparison of diagnostic efficacy of individual septic-screen markers (shown in table 8) in terms of overall specificity, PPV, NPV, and accuracy showed order of choice to be $-\mathrm{TLC}>\mathrm{ANC}>\mathrm{ITR}>\mathrm{mESR}>\mathrm{CRP}$ with least specificity (67\%) of CRP and mESR, although sensitivity wise order differed with ITR (75\%) and ESR (68\%) showing higher positivity than TLC/ANC (40-50\%) in preterms. Among the three most commonly obtained screening tests (TLC, CRP, ESR), mESR and TLC showed higher sensitivity, specificity, PPV, NPV, as well as accuracy; while most relied one i.e. CRP had the lowest sensitivity, specificity and overall diagnostic accuracy.

Table-8: Comparison of diagnostic efficacy of individual SS parameters.

\begin{tabular}{|c|c|c|c|c|c|}
\hline $\begin{array}{c}\text { Single SS } \\
\text { parameter +ve }\end{array}$ & Sensitivity & Specificity & $\begin{array}{c}\text { Positive } \\
\text { predictive } \\
\text { value }\end{array}$ & $\begin{array}{c}\text { Negative } \\
\text { predictive } \\
\text { value }\end{array}$ & Accuracy \\
\hline TLC & $50.00 \%$ & $97.53 \%$ & $91.67 \%$ & $78.22 \%$ & $80.80 \%$ \\
\hline CRP & $27.27 \%$ & $66.67 \%$ & $30.77 \%$ & $62.79 \%$ & $52.80 \%$ \\
\hline mESR & $68.18 \%$ & $66.67 \%$ & $52.63 \%$ & $79.41 \%$ & $67.20 \%$ \\
\hline IT RATIO & $75.00 \%$ & $75.31 \%$ & $62.26 \%$ & $84.72 \%$ & $66.68 \%$ \\
\hline ANC & $40.91 \%$ & $97.53 \%$ & $90.00 \%$ & $75.24 \%$ & $77.60 \%$ \\
\hline
\end{tabular}




\section{Original Research Article}

Paired combinations of at least 2 SS markers: Comparison of paired combinations of 2 SS-markers( as shown in table 9) in terms of overall diagnostic efficacy showed that all pairs of SS markers had better specificity (89-100\%, compared to individual markers) and high PPV (>88\%). Same time, analysing the sensitivity of 10 pairs of SS markers, the reducing order (with acceptable or comparable specificity): mESR+ITR $>$ TLC+ANC $>$ TLC+ITR $>$ ANC+ITR $>\mathrm{TLC}+\mathrm{mESR}>\mathrm{mESR}+\mathrm{CRP}>\mathrm{ANC}+>\mathrm{TLC}+\mathrm{CRP}>\mathrm{ANC}+\mathrm{CRP}>\mathrm{CRP}+\mathrm{ITR}$; suggests higher diagnostic value of pairs of simpler tests like mESR, TLC, ITR and ANC over lab-dependent test like CRP.

Table-9: Comparison of paired combinations of 2 parameters of sepsis- screen

\begin{tabular}{|l|c|c|c|c|c|}
\hline Pair of 2 SS markers & Sensitivity & Specificity & PPV & NPV & Accuracy \\
\hline TLC+CRP & $13.64 \%$ & $100 \%$ & $100 \%$ & $68.07 \%$ & $69.60 \%$ \\
\hline TLC+mESR & $22.73 \%$ & $98.77 \%$ & $90.91 \%$ & $70.18 \%$ & $72.00 \%$ \\
\hline TLC+IT RATIO & $31.82 \%$ & $100 \%$ & $100 \%$ & $72.97 \%$ & $76.00 \%$ \\
\hline TLC+ ANC & $40.91 \%$ & $88.89 \%$ & $66.67 \%$ & $73.47 \%$ & $72.00 \%$ \\
\hline CRP+ mESR & $20.45 \%$ & $96.30 \%$ & $75.00 \%$ & $69.03 \%$ & $69.60 \%$ \\
\hline CRP+ IT RATIO & $11.36 \%$ & $96.30 \%$ & $62.50 \%$ & $66.67 \%$ & $66.40 \%$ \\
\hline CRP + ANC & $11.36 \%$ & $100 \%$ & $100 \%$ & $67.50 \%$ & $68.80 \%$ \\
\hline mESR+IT RATIO & $52.27 \%$ & $96.30 \%$ & $88.46 \%$ & $78.79 \%$ & $80.80 \%$ \\
\hline mESR+ANC & $13.64 \%$ & $100 \%$ & $100 \%$ & $68.07 \%$ & $69.60 \%$ \\
\hline IT RATIO+ ANC & $27.27 \%$ & $100 \%$ & $100 \%$ & $71.68 \%$ & $74.40 \%$ \\
\hline
\end{tabular}

Combination of Multiple markers $\geq 3$ : Mean while, increasing number of SS markers in combinations to interprete as screen +ve result, for example a combo of three commonly obtained parameters e.g. (TLC+CRP+mESR) showed least sensitivity (6.82\%) in this study; though 100\% PPV, 66.39\% NPV and high (100\%) specificity may support its diagnostic value.

Local bacteriological profile of preterm sepsis amongst culture proven cases- Microbial profile of preterm sepsisin the present study setting revealed (as shown in table 10) that of total 44 culture positive cases, 15 (33.99\%) had Grampositive organisms including candida /fungal growth and $29(65.88 \%)$ had Gram-negative bacteria. Out of $2 / 3^{\text {rd }}$ gram negative growths, almost half were Klebsiella (15/29), and out of 15 Gram +ve organisms, 9 were staphaureus (60\%). Thus, among preterms with culture positive sepsis in this tertiary centremost common organisms were Klebsiella $>$ Staph aureus $>$ E. coli.

Table 10: Microbiological Culture-profile in preterms with proven sepsis

\begin{tabular}{|c|c|c|c|c|c|}
\hline $\begin{array}{c}\text { Gram +ve } \\
\text { Organism }\end{array}$ & $\begin{array}{c}\text { No. of cultures } \\
\text { grown }\end{array}$ & $\begin{array}{c}\text { \% of all } \\
\text { cultures }\end{array}$ & $\begin{array}{c}\text { Gram -ve } \\
\text { Organism }\end{array}$ & $\begin{array}{c}\text { No. of cultures } \\
\text { grown }\end{array}$ & $\begin{array}{c}\text { \% of all } \\
\text { cultures }\end{array}$ \\
\hline Staph aureus & 9 & 20.45 & 1. Klebsiella & 15 & 34.09 \\
\hline CONS & 4 & 9 & 2. E .Coli & 6 & 13.63 \\
\hline Candida & 2 & 4.54 & 3.Pseudomonas & 3 & 6.81 \\
\hline Enterococcus & 0 & 0 & 4.Acinetobacter & 3 & 6.81 \\
\hline & & & 5.Citrobactor & 1 & 2.27 \\
\hline & & & 6.Enterobacter & 1 & 2.27 \\
\hline Total & 15 & $33.99 \%$ & Total & 29 & 65.88 \\
\hline
\end{tabular}

\section{Discussion}

In this index study conducted at NICU of a tertiary hospital in middle India, majority of preterm $(<37$ weeks gestational age) newborns admitted with sepsis or its risk-factors were male (95\% of total 125 subjects), and almost $90 \%$ had more immaturity with GA $<35$ weeks. Such disproportionate sex-distribution in the study setting with good sex-ratio (>990 in Chhattisgarh)in the country, clearly indicates higher risk of neonatal sepsis in males amongst preterms. A significant proportion $(61 \%)$ had very low birth weight $(<1500 \mathrm{~g})$ and similarly, 58\% were outborn babies. All these neonatal and circumstantial factors might have posed additional 


\section{Original Research Article}

risks along with known maternal risk-factors for early onset sepsis (EOS) and might affect patient outcome. Punj et al [15] observed that during their study period, majority of septicaemia neonates were male $(63 \%)$, and $47 \%$ of babies were low birth weight. Similarly, Vinay BS et al [16] reported male and female ratio of 2:1 in their observational study, $68 \%$ babies were preterm and $70 \%$ were low birth weight. Hassan HR et al [17]also found that higher proportions of septic babies were male $(63.4 \%)$, preterm $(65.1 \%)$, low birth weight $(76.2 \%)$ and outborn (48\%).This concludes that prematurity or lower gestational age and low birth weight were the important physiological risk factors for neonatal sepsis.

In the present study, out of all sepsis-suspect and at risk cases, although risk factors of EOS ( $\geq 3$ of maternal plus neonatal risks) were documented in $47.2 \%$, clinically EOS was suspected or presumed to exist (at admission) in $83.2 \%$ cases. Irrespective of risk factors, amongst all preterm subjects, clinically presumed sepsis (either as EOS or LOS) developed in higher proportion $(92 \%)$. Well documented risk-factors of early neonatal sepsis definitely correlated with standard sepsis screen results (47\% vs $40 \% \mathrm{SS}+\mathrm{ve})$ as well as blood-culture (culture-positive sepsis was documented in $35 \%$ cases) in this study.

In a prior study, Vinay BS et al also found that majority of cases had early-onset sepsis (90\%), while $73 \%$ cases were septic screen positive and $80 \%$ cases were blood culture proven sepsis[16]. Higher blood culture positivity in their study might be due to inclusion of few contaminant growths. Similar to the present study, Zaka-ur-Rab $\mathrm{Z}$ et al [18] observed a nearly same proportion $(34.78 \%)$ of blood culture positive cases in studied population, though sepsis-screen was positive in much more $(69.57 \%)$ cases among subjects with clinically suspected sepsis, and even more (83.93\%) amongst cases with culture-proven true sepsis. Shams had Ali Z et al [19] found that amongst $46 \%$ culture positive (proven-sepsis) cases, 63\% were EOS and 37\% were LOS; while amongst culture negative cases, 34\% were EOS and $66 \%$ were LOS.

In present study, comparison of diagnostic efficacy of individual sepsis-screen (SS)-markers in terms of overall specificity, PPV, NPV, and accuracy (see table 7) suggested anorder of choice to be - TLC $>$ ANC $>$ ITR $>$ mESR $>$ CRP, although sensitivity wise order differed with ITR (75\%) and ESR(68\%) showing higher positivity than TLC/ANC (40-50\%) and CRP (27\%)in preterm sepsis. Lacour AG et al also suggested that even single markers like CRP, and procalcitoninaid the clinician in the initiation and stopping of antibiotic therapy [20].

In contrast to the present study, Zaka-ur-Rab $\mathrm{Z}$ et al found that amongst individual markers of sepsis screen, CRP had good diagnostic utility with highest sensitivity (82\%) and specificity (89\%)[18]. Lakhey A et al found that CRP $(78 \%)$ and IT ratio $(73 \%)$ had highest sensitivity[21].

In the present study, out of three most commonly obtained screening tests in practice (TLC, CRP, ESR), mESR and TLC showed higher sensitivity, specificity, PPV, NPV as well as overall diagnostic accuracy compared to CRP. Chandrakoshi et al found that absolute neutrophil count(ANC) has higher sensitivity and specificity than total WBC count[22]. Practically, $\mathrm{CBC}$ alone delivers three of five screen parameters i.e. TLC, ANC, as well as ITR, if good side-lab microscopy is available and mESR can also be obtained bedside by glass-capillary method.

In present study, comparison of paired combinations of 2 SS-markers (as shown above in table 8) in terms of overall diagnostic efficacy showed that all 10 pairs of five SS markers had better specificity (89-100\%), and high PPV (>88\%) compared to individual markers. The reducing order of sensitivity (being asmESR+ITR $>$ $\mathrm{TLC}+\mathrm{ANC}>\mathrm{TLC}+\mathrm{ITR}>\mathrm{ANC}+\mathrm{ITR}>\mathrm{TLC}+\mathrm{mESR}>$ $\mathrm{mESR}+\mathrm{CRP}>\mathrm{ANC}+>\mathrm{TLC}+\mathrm{CRP}>\mathrm{ANC}+\mathrm{CRP}>$ CRP+ITR) suggests higher diagnostic value of pairs of simpler tests like mESR, TLC, ITR and ANC over labdependent test like CRP. Meanwhile, combination of multiple or $\geq 3$ tests, for example, combo of three commonly obtained parameters e.g. (TLC+CRP+ mESR) showed least sensitivity $(6.82 \%)$, although specificity was $100 \%$.

Vinay BS et al showed that a septic screen (positive with $\geq 2$ markers) had sensitivity of $77 \%$, specificity of $41 \%$, PPV of $84 \%$ and NPV of $31 \%$, when blood culture is considered as gold standard to detect neonatal sepsis[16]. Comparable to the present study, Zaka-ur$\mathrm{Rab} \mathrm{Z}$ et alfound thata combination of CRP plus I/T ratio had the highest sensitivity (79\%), specificity (100\%), PPV (100\%) and NPV (89.47\%) [18]. Punyashety $\mathrm{KB}$ et al reported that haematological parameters like TLC, I/T ratio and CRP showed high sensitivity, specificity, PPV and NPV as individual marker as well as in combinations and results were promising when tests were evaluated in combinations using haematological scoring system [23]. Lazarus 
Monica at el also found that if multiple $>2$ of the tests are obtained, sensitivity and negative predictive value of the screening tool increased to more than $90 \%$ [24]. So, both considering single and too many markers $(\geq 3)$ being positive to indicate probable sepsis would not be suitable for ideal/standard screening tool, especially in preterm newborns, those might not be able to mount all inflammatory response and in same time-frame when compared with term neonates.

Limitation of this study: The study is not a novel one, rather just a validating step to confirm and support the role as well as utility of available sepsis-screening tests even in preterm newborns. This favours adequacy of minimum of any two easily obtained test-markers, but advocates good clinico-logical interpretation of these in clinical practice.

\section{Conclusion}

Sepsis amongst preterm newborns was although observed more in very low birth weight, more premature, outborn, and male sex; neonates with additional maternal risk factors with/without clinical presentation of sepsis had high chance of having sepsisscreen +ve 'probable sepsis' as well as culture provensepsis. Comparison of diagnostic efficacy of individual SS-markers in terms of overall diagnostic accuracy suggested an order of choice to be - TLC $>$ ANC $>$ ITR $>$ mESR $>$ CRP, but better diagnostic utility was seen with paired or $\geq 2$ positive markers due to higher specificity, sensitivity, PPV and NPV, even in preterms.

\section{What this study adds to existing knowledge?}

As role of standard sepsis-screening methods is already known in neonatal sepsis, this study conducted on selective preterm subpopulation favours the acceptable diagnostic efficacy of minimum of any 2 of $5 \mathrm{SS}$ markers. Timely screening with simple and minimum tests like CBC with microscopy (for ANC \& ITR, with TLC) and/orm ESR, can also add high diagnostic probability to our clinical suspicion of sepsis; and even preterms with variable presentations can be managed early without waiting for complex tests or culture reports.

\section{Author's contributions:}

Dr. Pratima Beck: Conceptualized and designed the study.

Dr. Santosh Kumar Rathia: Conceptualized and designed the study, data analysis, manuscript writing.
Dr. Sharja Phuljhele: Conceptualized and designed the study, provided department support.

Dr. Swati Kurrey: Protocol writing, data collection, data analysis and manuscript writing

Funding: Nil, Conflict of interest: None initiated, Permission from IRB: Yes

\section{References}

1. Bang AT, Bang RA, Baitule SB, Reddy $\mathrm{MH}$, Deshmukh MD. Effect of home-based neonatal care and management of sepsis on neonatal mortality: field trial in rural India. Lancet. 1999;354(9194):1955-61. doi:10. 1016/S0140-6736(99)03046-9

2. Stoll BJ. The global impact of neonatal infection. Clin Perinatol. 1997;24(1):1-21.doi:https://doi.org/ 10. 1016/ S0095-5108(18)30181-7

3. Moore MR, Schrag SJ, Schuchat A. Effects of intrapartum antimicrobial prophylaxis for prevention of group-B-streptococcal disease on the incidence and ecology of early-onset neonatal sepsis. Lancet Infect Dis. 2003; 3(4):201-13. doi: https://doi.org/ 10.1016/ S1473- 3099(03)00577-2

4. Stoll BJ, Hansen N, Fanaroff AA, Wright LL, Carlo WA, Ehrenkranz RA, et al. Late-onset sepsis in very low birth weight neonates: the experience of the NICHD Neonatal Research Network. Pediatrics. 2002; 110 (2 Pt 1):285-91. doi:10.1542/peds.110.2.285

5. Rodwell RL, Leslie AL, Tudehope DI. Early diagnosis of neonatal sepsis using a hematologic scoring system. J Pediatr. 1988;112(5):761-7. doi:10. 1016/ s0022-3476(88)80699-1

6. Ng PC. Diagnostic markers of infection in neonates. Arch Dis Child Fetal Neonatal Ed. 2004;89(3):F229-35. doi:10.1136/adc.2002.023838

7.GotoffSP,Behrman RE.Neonatal septicemia. J Pediatr. 1970;76(1):142-53. doi10.1016/s0022-3476(70)80148-2

8. Sankar MJ, Agarwal R, Deorari AK, Paul VK. Sepsis in the newborn. Indian J Pediatr. 2008;75(3):261-6. doi:10.1007/s12098-008-0056-Z

9. Mishra UK, Jacobs SE, Doyle LW, Garland SM. Newer approaches to the diagnosis of early onset neonatal sepsis. Arch Dis Childhood: Fetal and Neonatal Ed. 2006; 91(3):F208-F212.doi: 10.1136/ adc. 2004. 064188 


\section{Original Research Article}

10. Singh M, Narang A, Bhakoo ON. Predictive perinatal score in the diagnosis of neonatal sepsis. J Trop Pediatr. 1994;40(6):365-8. doi: 10.1093/tropej/ 40. 6. 365 .

11. Polinski C. The value of the white blood cell count and differential in the prediction of neonatal sepsis. Neonatal Netw. 1996;15(7):13-23.

12. Da Silva O, Ohlsson A, Kenyon C. Accuracy of leukocyte indices and $\mathrm{C}$-reactive protein for diagnosis of neonatal sepsis: a critical review. Pediatr Infect Dis J. 1995; 14(5):362-6.

13. Manroe BL, Weinberg AG, Rosenfeld CR, Browne R. The neonatal blood count in health and disease. I. Reference values for neutrophilic cells. J Pediatr. 1979;95(1):89-98. doi:10.1016/s0022-3476(79)80096-7

14. Mouzinho A, Rosenfeld CR, Sánchez PJ, Risser R. Revised reference ranges for circulating neutrophils in very-low-birth-weight neonates. Pediatr. 1994; 94(1): 76-82.

15. Punj A, Kapila S, Goel SP, Prakash A, Dubey A. Predictability Of Sepsis On The Basis Of Clinical Features. Int JMedSci Clin Invent.2015;2(9):1306-1311

16. Vinay BS, Girish GN, Adhikari S, Hugara S. Evaluation of septic screen as a diagnostic tool for neonatal sepsis in a tertiary hospital at Mysore. Sch J App Med Sci. 2015;3(2G):1005-1010.

17. Hassan HR, Gohil JR, Desai R, Mehta RR, Chaudhary VP. Correlation of blood culture results with the sepsis score and sepsis screen in the diagnosis of early-onset neonatal septicemia. J Clinic Neonatol. 2016; 5(3):193.
18. Zaka-ur-Rab Z, Kar M, Gupta V, Kalra BP, Kafil Akhtar K. Evaluation of laboratory markers of sepsis screen in the diagnosis of early onset neonatal septicemia. Int J Contemp Pediatr 2016;3(4):11441149.doi: http://dx.doi.org/10.18203/2349-3291.ijcp 20163146

19. Ali ZS, Ghodke BA, Deka AB. Sepsis screen parameters as a tool for diagnosis of neonatal sepsis. J Evol Med Dental Sci. 2017;6(79):5584-9.

20. Lacour AG, Gervaix A, Zamora SA, Vadas L, Lombard PR, Dayer JM, et al. Procalcitonin, IL-6, IL-8, IL-1 receptor antagonist and C-reactive protein as identificators of serious bacterial infections in children with fever without localising signs. Eur J Pediatr. 2001; 160(2):95-100.

21. Lakhey A, Shakya H. Role of sepsis screening in early diagnosis of neonatal sepsis. J Pathol Nepal. 2017; 7(1):1103-10. doi: https://doi.org/10.3126/jpn. v7i1. 16944

22. Chandrakoshi G, Gond SK. Role of various hematological parameters in diagnosis of clinically suspected cases of neonatal septicemia. Int J Biol Med Res. 2018; 9(3):6389-6392.

23. Punyashetty KB, Patil T. Interpretation of haematological parameters in neonates at risk for sepsis. J Evid Based Med Health. 2017;49(3):2492-2496.

24. Monica L, Riti JS, Amit BK. Role of Sepsis Screen Parameters in Early Diagnosis of Neonatal Septicemia. Int J Curr Microbiol Appl Sci 2018; 7: 2410-2419. doi: https://doi.org/10.20546/ijcmas.2018.701.290

\section{How to cite this article?}

Beck P, Rathia S.K, Phuljhele S, Kurrey S. Diagnostic efficacy of sepsis-screen parameters (individual and combined) compared to blood culture and clinico-etiological profile of neonatal sepsis in preterm newborns. Int J Pediatr Res. 2019; 6(07):372-380.doi:10. 17511/ijpr.2019.i07.10 